\title{
Characteristics of circulating tumor cells in organ metastases, prognosis, and T lymphocyte mediated immune response
}

This article was published in the following Dove Press journal:

OncoTargets and Therapy

4 May 2017

Number of times this article has been viewed

\author{
Wen-Wen Sun ${ }^{1, *}$ \\ Zhi-Hong $\mathrm{Xu}^{1}{ }^{1, *}$ \\ Peng Lian' \\ Bei-Li Gao ${ }^{2}$ \\ Jia-An $\mathrm{Hu}^{\prime}$ \\ 'Department of Geriatrics, \\ 2Department of Respiration, Ruijin \\ Hospital, Shanghai Jiaotong University, \\ Shanghai, People's Republic of China \\ *These authors contributed equally \\ to this work
}

Correspondence: Jia-An Hu

Department of Geriatrics, Ruijin Hospital, Shanghai Jiaotong University, Shanghai 200025, People's Republic of China

Tel +86 I 360 I 7370 I4

Fax +862154198967

Email hujiaan@aliyun.com

Bei-Li Gao

Department of Respiration, Ruijin Hospital, Shanghai Jiaotong University, Shanghai 200025, People's Republic of China

Tel $+86 \quad 1360 \quad 1772598$

Fax +862154198967

Email beiligao@aliyun.com
Abstract: Circulating tumor cells (CTCs) possess profound influence on tumor metastases and disease progression. This study aimed to investigate the correlation of CTCs with clinical characteristics and T-cell immunity, and to explore whether CTCs and the subpopulations can serve as an independent prognostic factor in advanced non-small cell lung cancer (NSCLC). A prospective study was conducted in late stages of NSCLC patients. The levels of overall CTCs and the three subpopulation CTCs were enumerated using the CanPatrol ${ }^{\text {TM }}$ CTC enrichment system. The information about the patients which included the clinical characteristics, survival status at the 200th day postdiagnosis, and the levels of T cells was collected. Mann-Whitney $U$ test, Kruskal-Wallis $H$ test, Cox regression, and Spearman's rank correlation coefficient were the statistical methods used in this study. We detected CTCs in 27 of the 31 eligible patients; the level of epithelialmesenchymal circulating tumor cells (EMCTCs) was higher than that of epithelial circulating tumor cells and that of mesenchymal circulating tumor cells (MCTCs) in the majority of NSCLC patients. Organ metastases were positively associated with the levels of overall CTCs, EMCTCs, and MCTCs $(P<0.05)$. EMCTCs and MCTCs were associated with worse clinical outcomes. Additionally, the levels of EMCTCs were negatively associated with the levels of $\mathrm{CD}^{+} \mathrm{T}$ cells ( $P=0.01)$ and $\mathrm{CD}^{+} \mathrm{T}$ cells $(P=0.04)$. In conclusion, the levels of $\mathrm{CTCs}$ were positively associated with organ metastases, particularly bone metastases, but were negatively associated with T-cell levels. The levels of EMCTCs and MCTCs had negative prognostic value.

Keywords: clinical trial, non-small cell lung cancer, circulating tumor cells, organ metastases, prognosis, lymphocyte $\mathrm{T}$ immune system

\section{Introduction}

According to the GLOBOCAN 2012 statistical analysis, the incidence of and mortality from lung cancer are higher compared with those from other forms of cancer, with the exception of nonmelanoma skin cancer. ${ }^{1}$ Metastasis is the leading cause of death from lung cancer. ${ }^{2}$ Circulating tumor cells (CTCs) can provide real-time biopsy specimens for studying the biologic behavior of tumor metastasis. ${ }^{3}$ CTCs refer to the tumor cells which actively or passively detach from a solid primary or secondary neoplasm and enter the peripheral circulation. On the basis of morphologic features, CTCs are divided into CTC clusters and single CTCs. ${ }^{4}$ Furthermore, single CTCs are generally classified into three subpopulations including epithelial circulating tumor cells (ECTCs), epithelial-mesenchymal circulating tumor cells (EMCTCs), and mesenchymal circulating tumor cells (MCTCs). ${ }^{4}$

CTCs are continuously under attacks from unfavorable tumor microenvironment which contains the immune surveillance system. T-cell-mediated immunity 
plays a pivotal role in cancer metastasis. Specific T-cell immunoregulatory mechanisms can create an immunologically permissive environment for tumor colonization. ${ }^{5,6}$ In the research of breast cancer, Mego et $\mathrm{al}^{7}$ had found that patients with CTCs had abnormalities in T-cell-mediated immunity that could potentially initiate and impact the dissemination of tumor cells. Mittal et $\mathrm{al}^{8}$ displayed an overall review of cancer immunoediting and its three phrases - elimination, equilibrium, and escape - which was helpful to understand the positive, negative, or null effects that the T-cell immune system has on tumor development.

As a minimally invasive and multifunctional biomarker, the clinical relevance of CTCs in the tumor node metastasis stage system and prognosis is well established..$^{9-12}$ Nevertheless, the significance of CTC subpopulations in the overall survival, organ metastases, and immune system has rarely been studied in patients with non-small cell lung cancer (NSCLC).

This study explored the correlation between different subpopulations of CTCs and organ metastases, and analyzed the prognostic significance of total CTCs (TCTCs) and subpopulations of CTCs. We also discussed the relationship between T-cell immunity and different CTC phenotypes, and further elucidated the role of TCTCs and CTC subpopulations in prognosis, organ metastases, and the immune system. This study should be helpful to prevent the occurrence of metastasizing-homing-remetastasizing and develop more effective therapeutics.

\section{Materials and methods Patients}

This clinical trial was open to individuals not $<18$ years old who were diagnosed with clinical stage III or IV lung cancer; pathologic types were restricted to adenocarcinoma or adenosquamous carcinoma. The Eastern Cooperative Oncology Group performance status score of the patients ranged from 0 to 2 . The exclusion criteria were: the patients who had taken other therapeutics other than ALIMTA plus carboplatin or the first-generation targeted drugs previously, the patients belonging to clinical stage I or II, and the patients who also had another malignancy or severe systemic disease. This study was conducted in accordance with the Declaration of Helsinki and was approved by the Ethics Committee of Shanghai Jiaotong University. Written informed consent was obtained from all participants.

\section{Study design}

This was an open-label, single-arm, single-center, prospective study. Patients who conformed to the inclusion criteria underwent a one-time collection of CTCs. The primary endpoint of this study was the overall survival (OS) at the 200th day postdiagnosis. Patients with a mutation of epidermal growth factor receptor (EGFR) on exon 19 or 21 were administered first-generation EGFR-targeted agents. However, other patients with better health condition were treated with chemotherapeutic agents (Eli Lilly, Indianapolis, IN, USA); also, patients with deteriorated health condition chose to taken first-generation EGFR-targeted agents. All the information, including the general characteristics of the patients, metastasis-related characteristics, and T-cell levels, was utilized to assess OS and to analyze the correlation with CTCs. Organ metastases were classified into extra-lung metastases and intra-lung metastases. Bone metastases were divided into cranial metastases, vertebral metastases, truncal bone (with the exclusion of vertebral) metastases, and metastases in the extremities. The details are listed in Figure 1.

Each patient underwent chest computed tomography (CT), bone scan, brain magnetic resonance, abdominal ultrasound, or whole-body positron emission tomography/CT within 1 month of CTC detection. General information (age, gender, family history of cancer, smoking history, whether in first treatment) and disease-related characteristics (pathologic type, tumor location, tumor stage, T stage, $\mathrm{M}$ stage, $\mathrm{N}$ stage, and organ metastases) were collected from all participants. As CTCs are closely correlated with metastatic properties, $\mathrm{M}$ stage was categorized in detail to explore the correlation of CTC levels with different organ metastases and to ascertain which organs the CTCs predominantly home into in patients with advanced NSCLC. This study used the eighth edition of the International Association for the Study of Lung Cancer (IASLC) staging system of lung cancer. ${ }^{13}$

\section{Sample collection}

Before conducting therapy at the Department of Respiration in Ruijin Hospital, 5-6 mL peripheral venous blood was collected in ethylenediaminetetraacetic acid (EDTA) tubes from each of the enrolled patients; EDTA tubes were agitated gently several times, then the blood was transferred from the EDTA tube to a storage tube and again gently agitated several times. The storage tube was placed at room temperature $\left(15^{\circ} \mathrm{C}-30^{\circ} \mathrm{C}\right)$ for 30 minutes before being stored at $2^{\circ} \mathrm{C}-8^{\circ} \mathrm{C}$. Samples were transported to the test center and CTC detection was performed using the CanPatrol ${ }^{\mathrm{TM}}$ system (SurExam, Guangzhou, People's Republic of China) within 1 week of collection.

\section{Detection of CTCs}

CTCs were detected with the CanPatrol CTC enrichment system using filtration and tricolor RNA hybridization in situ (RNA-ISH). 


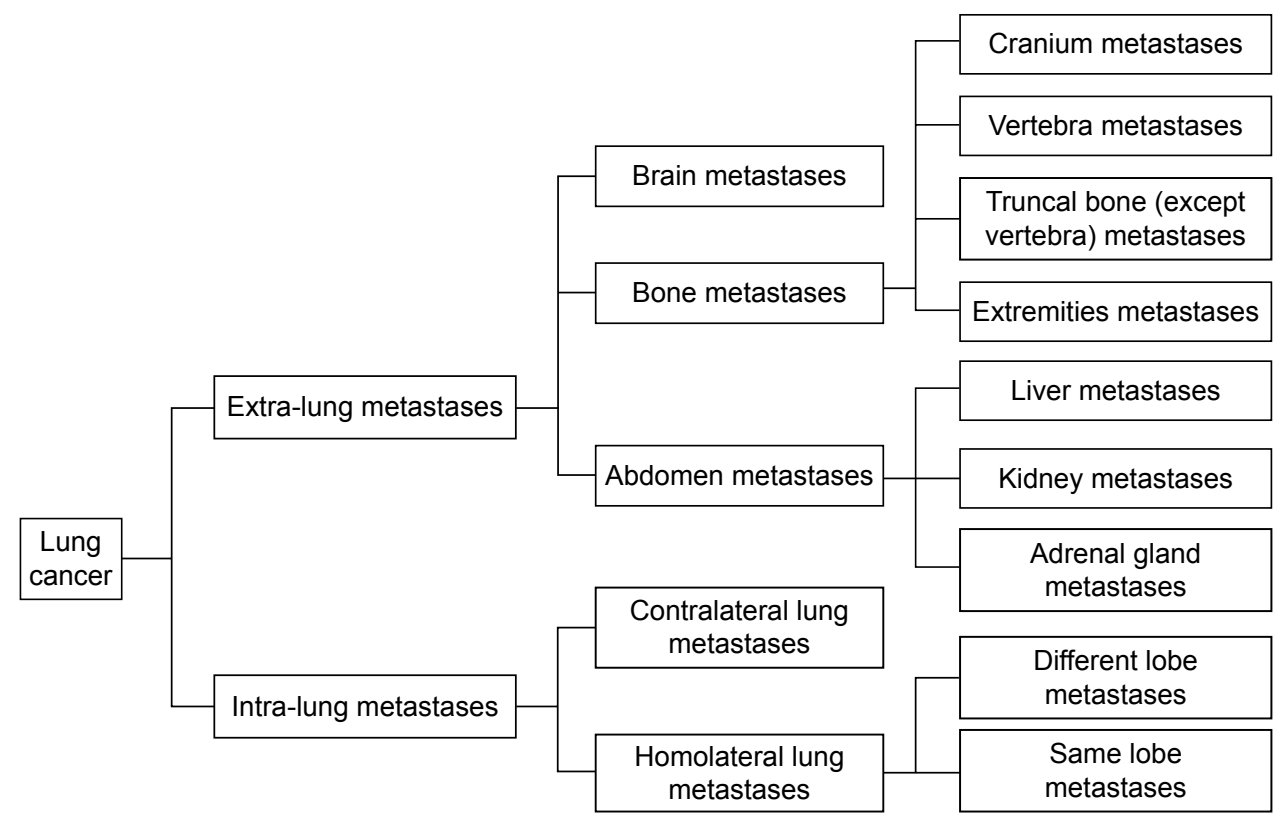

Figure I Organ metastases.

The filtration system was based on the size of the cells. Before filtration, erythrocytes were removed from the samples using the red blood cell lysis solution (Sigma-Aldrich Co., St Louis, MO, USA); then, the remaining cells were resuspended in phosphate-buffered saline (PBS; SigmaAldrich Co.) with $4 \%$ formaldehyde (Sigma-Aldrich Co.) for 5 minutes. Each cell suspension was then transferred to a filtration tube (SurExam). The filtration system was composed of a membrane with $8 \mu \mathrm{m}$ diameter pores (EMD Millipore, Billerica, MA, USA), a manifold vacuum plate with valve settings (SurExam), an E-Z 96 vacuum manifold (Omega, Norcross, GA, USA), and a vacuum pump (Auto Science, Tianjin, People's Republic of China). Filtration was initiated at $0.08 \mathrm{MPa}$, followed by tricolor RNA-ISH based on branched DNA signal amplification.

Tricolor RNA-ISH was conducted in a $24-$ well plate (Corning Incorporated, Corning, NY, USA) after the membrane with $8 \mu \mathrm{m}$ diameter pores was removed from the filtration tube. During tricolor RNA-ISH, two categories of probes, capture probes and signal amplification probes, were used. The cells on the membrane were treated with protease $100 \mu \mathrm{L} /$ well (Qiagen, Hilden, Germany), and capture probes were applied for $3 \mathrm{~h}$ at $40^{\circ} \mathrm{C}$ to detect targeted sequences for hybridization. The capture probes can detect epithelial biomarkers including epithelial cell adhesion molecules (EpCAMs) and cytokeratin (CK) 8/18/19, mesenchymal biomarkers (vimentin and Twist), and the leukocyte biomarker CD45. ${ }^{14}$ After washing three times with $1,000 \mu \mathrm{L}$ wash buffer (Sigma-Aldrich Co.), the signal amplification step was performed with three kinds of amplification probes: preamplifier sequence, amplifier sequence, and a label probe conjugated to a fluorescent dye. ${ }^{15}$ All the sequences were manufactured by Thermo Fisher Scientific (Waltham, MA, USA). The captured samples were incubated with preamplifier solution (Sigma-Aldrich Co.) $100 \mu \mathrm{L} /$ well at $40^{\circ} \mathrm{C}$ for 30 minutes. Then, the membranes were washed three times with $1,000 \mu \mathrm{L}$ wash buffer and incubated with amplifier solution (Sigma-Aldrich Co.) $100 \mu \mathrm{L} /$ well at $40^{\circ} \mathrm{C}$ for 30 minutes. Finally, the label probes conjugated to Alexa Fluor 594 (for epithelial biomarkers), Alexa Fluor 488 (for mesenchymal biomarkers), and Alexa Fluor 647 (for the leukocyte biomarker) were added and incubated for 30 minutes at $40^{\circ} \mathrm{C}$ for hybridization with the branched DNA molecule. After washing with wash buffer, the cells were stained with 4',6-diamidino-2-phenylindole (Sigma-Aldrich Co.) for 5 minutes, then observed with a fluorescence microscope using a $40 \times$ objective and a $10 \times$ ocular lense (Olympus BX53; Olympus Corporation, Tokyo, Japan).

\section{Collection and detection of $\mathrm{T}$ cells}

Patients who were previously untreated were assessed for the levels of $\mathrm{CD}^{+} \mathrm{T}$ cells, $\mathrm{CD} 4^{+} \mathrm{T}$ cells, and $\mathrm{CD} 8^{+} \mathrm{T}$ cells. Two milliliters of peripheral venous blood was collected in an EDTA tube and sent to the clinical laboratory within 4 hours of collection, followed by T-cell detection. Monoclonal antibody with fluorescence $(5 \mu \mathrm{L})$ was added to $30 \mu \mathrm{L}$ blood; $200 \mu \mathrm{L}$ hemolysin was added 15-20 minutes later. After 10-15 minutes, $200 \mu \mathrm{L}$ PBS was added and then the 
samples were examined using flow cytometry (BD FACS CantoII Flow Cytometry System). Before detection, BD FACS 7-Color Setup Beads (335775) were applied to adjust detector voltages and set fluorescence compensation.

\section{Statistical analysis}

SPSS17.0 IBM software was used to conduct statistical analysis. Nonparametric tests including Mann-Whitney $U$ test (for binary variables) and Kruskal-Wallis $H$ test (for ranked data) were used to assess the predictive power of the levels of TCTCs and subpopulations of CTCs in different clinical pathologic characteristics $(\alpha=0.05, P<0.05)$. Median and interquartile range were used to describe variability. The significance of the TCTCs, the three CTC subpopulations, the clinical characteristics, and the therapeutic regimens to OS on 200th day was analyzed using the univariate Cox regression analysis $(\alpha=0.05, P<0.05)$. Significant parameters in univariate Cox regression analysis were subjected to multivariate Cox regression analysis. Bivariate correlation analysis was performed to study the association between the levels of CTCs and T-cell-mediated immunity $(P<0.05)$.

\section{Results}

\section{General characteristics of the enrolled patients with NSCLC}

From October 2014 to September 2015, thirty-one eligible patients at the Respiratory Department of Ruijin Hospital were enrolled in this study and provided informed consent. There were 17 women, 21 patients $<65$ years old, three patients who had a family history of cancer, 23 participants without previous treatment, and eight participants with targeted drug therapy or chemotherapy. During the follow-up periods, ten patients with the mutation of L858R or exon 19 deletion were treated with first-generation targeted drug therapies.

\section{Characteristics of each subpopulation of CTCs}

Three subpopulations of CTCs (ECTCs, EMCTCs, and MCTCs) were isolated from the peripheral venous blood using the CanPatrol CTC platform. ECTCs that had only epithelial molecular markers (EpCAM and CK 8/18/19) were stained with red immunofluorescence; MCTCs possessing mesenchymal molecular markers (vimentin and Twist) were stained with green immunofluorescence; and EMCTCs with the two kinds of molecular markers were stained by green and red immunofluorescence (Figure 2). CTCs were detected in 27 out of 31 stage III or IV NSCLC patients.
Sixteen out of 27 patients had higher levels of EMCTCs than ECTCs and MCTCs; three patients had equivalent levels of EMCTCs to those of ECTCs and MCTCs (Figure 3). There were 19 patients whose TCTCs were more than 4/5-6 mL peripheral venous blood and eight patients whose TCTCs were more than $10 / 5-6 \mathrm{~mL}$ peripheral venous blood.

\section{Association between the levels of CTCs and general characteristics of patients with NSCLC}

The levels of TCTCs in patients who had undergone treatment were significantly greater than those in untreated patients (treated patients: 9, [6.25, 17.75]; untreated patients: $4,[1,8] ; P=0.019)$. EMCTC levels were also significantly higher in treated patients (treated patients: 7, $[6,10.25]$; untreated patients: 2, $[0,4] ; P=0.004)$. ECTC levels showed statistical difference with respect to age ( $\geq 65$ years old: 2 , $[0,4.5] ;<65$ years old: $0,[0,1] ; P=0.044)$. However, no statistically significant associations of TCTCs and the three subpopulations were discovered with gender, smoking status, or family history of cancer (Table 1).

\section{Association between CTCs and the staging system}

TCTCs did not show statistical significance in tumor stage. However, ECTCs showed statistical difference in tumor stage (stage III: $0,[0,0]$; stage IV: $1,[0,2] ; P=0.020$ ), in the M stage (M0: 0, [0, 0]; M1a: 0, [0, 0]; M1c: 1, [0, 2.5]; $P=0.017$ ), and in the N stage (N0: $1,[0,2] ; \mathrm{N} 2: 0,[0,0.25]$; N3: $1,[0,4.5] ; P=0.026)$. In the $M$ stage, the differences mainly originated from comparison between $\mathrm{M} 0$ and M1a and between M0 and M1c. In contrast, TCTCs and the three subpopulations showed no statistical significance with respect to the pathologic type, tumor location, and T stage (Table 2).

\section{Relationship of CTCs with organ metastases}

Statistical analysis of CTCs regarding organ metastases demonstrated that the number of organ metastases was significantly correlated with the levels of TCTCs (metastases $<3$ : 5, $[0.5,7.5]$; metastases $\geq 3: 11,[3.25,27.25] ; P=0.028)$, EMCTCs (metastases $<3: 3,[0,6]$; metastases $\geq 3: 7,[1.25$, 13.25]; $P=0.040$ ), and MCTCs (metastases $<3: 0$, [0,0]; metastases $\geq 3: 1,[0,3] ; P=0.023)$. ECTCs showed statistical association with extrapulmonary metastases (yes: 1 , $[0,2.5]$; no: $0,[0,0] ; P=0.004)$. Significant association was also observed between the severity of pleural effusion and 

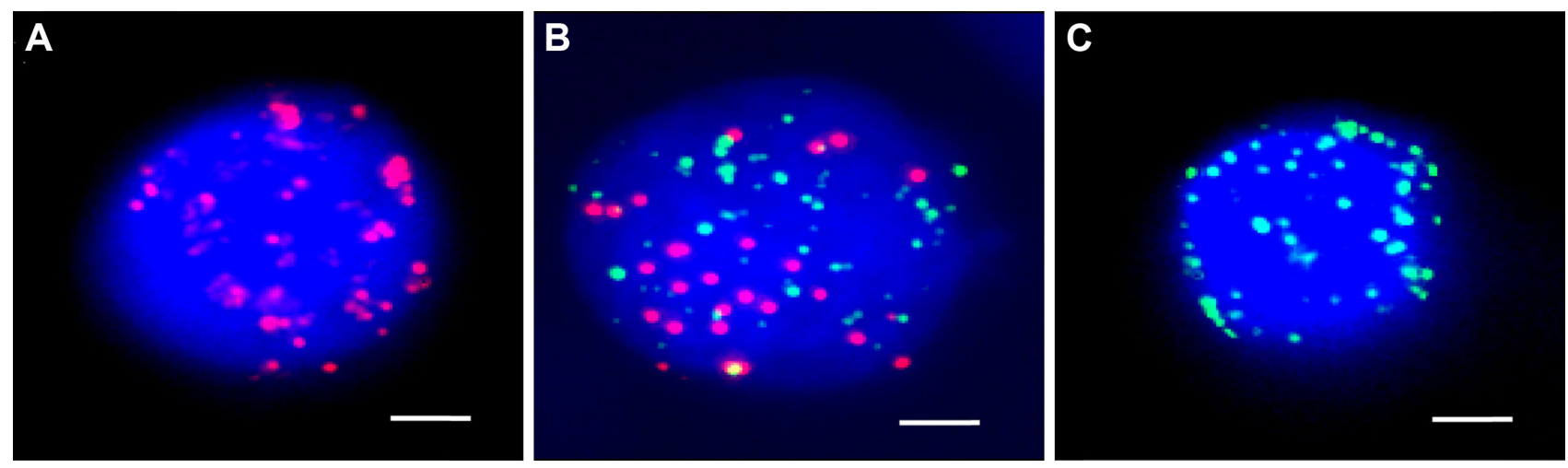

Figure 2 (A) ECTCs possessing only epithelial molecular markers (EPCAM and cytokeratin 8/18/19) were stained with red immunofluorescence. (B) EMCTCs having both epithelial and mesenchymal molecular markers were stained with both green and red immunofluorescent dyes. (C) MCTCs occupying only mesenchymal molecular markers (vimentin and Twist) were stained with green immunofluorescence. (Bars $=2.5 \mu \mathrm{m}$ ).

Abbreviations: ECTCs, epithelial circulating tumor cells; EMCTCs, epithelial-mesenchymal circulating tumor cells; EpCAM, epithelial cell adhesion molecule; MCTCs, mesenchymal circulating tumor cells.

MCTCs (yes: 3, [0, 4]; no: 0, [0, 0]; $P=0.009$; Table 3). Patients with abdominal organ metastases (paranephrous, kidney, and liver), pulmonary metastases, or brain metastases displayed no significant association with the levels of TCTCs and those of the three subpopulations.

\section{Relationship between CTCs and bone metastases}

In this study, bone was found to be the favorable extrapulmonary organ for the dissemination of neoplastic cells. Twenty out of 31 patients had metastatic bone lesions, and 19 out of these
20 patients were detected with CTCs. Although the levels of TCTCs did not show statistical difference in bone metastases, ECTC levels showed a statistically significant difference (yes: $1,[0,3.5]$; no: $0,[0,0.5] ; P=0.019$ ). Among the patients with bone metastases, 15 patients had metastases in the vertebrae, and 14 out of these 15 patients were detected with CTCs. The levels of ECTCs showed statistical significance with respect to vertebral metastases (yes: $1,[0,2]$; no: $0,[0,1]$; $P=0.042$ ). Levels of TCTCs and of CTC subtypes did not show statistical significance in the extremities metastases, truncal bone metastases, or the cranium metastases (Table 3).

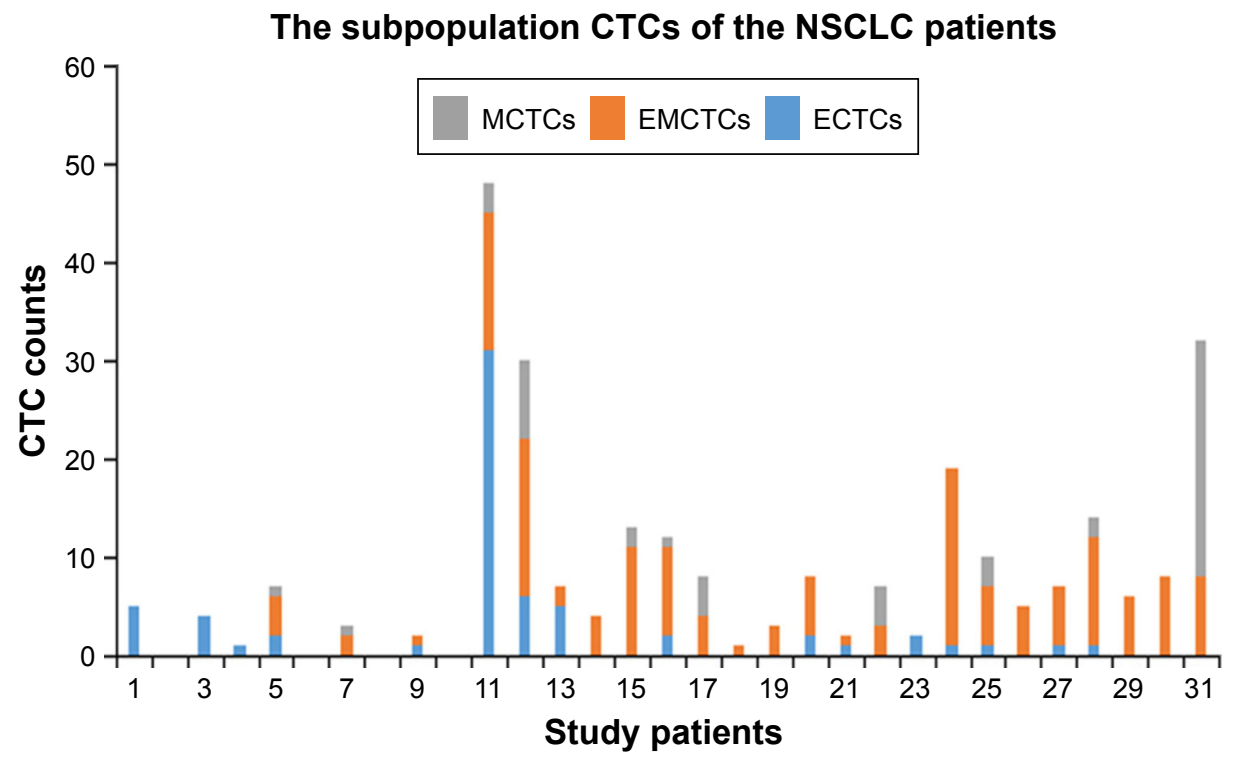

Figure 3 Twenty-seven out of 31 patients were detected with CTCs. The levels of EMCTCs predominated within the three CTC subpopulations among I6 patients and the levels of EMCTCs were equivalent to ECTCs count or MCTCs count among three patients.

Abbreviations: CTCs, circulating tumor cells; ECTCs, epithelial circulating tumor cells; EMCTCs, epithelial-mesenchymal circulating tumor cells; MCTCs, mesenchymal circulating tumor cells; NSCLC, non-small cell lung cancer. 
Table I CTCs and the general characteristics

\begin{tabular}{|c|c|c|c|c|c|}
\hline Characteristics & Number & TCTCs & ECTCs & EMCTCs & MCTCs \\
\hline \multicolumn{6}{|l|}{ Age (years) } \\
\hline$<65$ & 21 & $5(1.5,10)$ & $0(0,1)^{*}$ & $3(0.5,8)$ & $0(0,1)$ \\
\hline$\geq 65$ & 10 & $7(3.5,15)$ & $2(0,4.5)^{*}$ & $4(0,8)$ & $0.5(0,3.25)$ \\
\hline \multicolumn{6}{|l|}{ Gender } \\
\hline Female & 17 & $5(2.5,7.5)$ & $0(0,4.5)$ & $3(0,6)$ & $0(0,2.5)$ \\
\hline Male & 14 & $7.5(1.5,12.5)$ & $\mathrm{I}(0, \mathrm{I} .25)$ & $5(0.75,8.25)$ & $0(0,1.25)$ \\
\hline \multicolumn{6}{|l|}{ Smoking } \\
\hline Yes & 11 & $4(0,12)$ & $0(0,1)$ & $4(0,8)$ & $0(0,0)$ \\
\hline No & 20 & $6.5(3,9.5)$ & $\mathrm{I}(0,3.5)$ & $3.5(0.25,6.0)$ & $0(0,2.75)$ \\
\hline \multicolumn{6}{|c|}{ Family history of cancer } \\
\hline Yes & 3 & $12(2,19)$ & $I(I, 2)$ & $9(1,18)$ & $0(0,1)$ \\
\hline No & 28 & $5.5(2,8)$ & $0(0,2)$ & $3.5(0,6)$ & $0(0,2)$ \\
\hline \multicolumn{6}{|l|}{ Untreated } \\
\hline Yes & 23 & $4(1,8)^{*}$ & $I(0,2)$ & $2(0,4)^{*}$ & $0(0,1)$ \\
\hline No & 8 & $9(6.25,17.75)^{*}$ & $0.5(0,1)$ & $7(6,10.25)^{*}$ & $0(0,2.75)$ \\
\hline
\end{tabular}

Notes: $* P<0.05$; statistical description: median (figures outside the parentheses) and interquartile range (figures in the parentheses).

Abbreviations: CTCs, circulating tumor cells; ECTCs, epithelial circulating tumor cells; EMCTCs, epithelial-mesenchymal circulating tumor cells; MCTCs, mesenchymal circulating tumor cells; TCTCs, total CTCs.

\section{Prognostic value of CTCs}

The OS analysis was conducted after the follow-up of 200 days. Univariate Cox regression survival analysis demonstrated that age was a risk factor to OS (HR 3.939; $95 \%$ confidence index [CI], 1.190-12.971; $P=0.027)$. Additionally, the number of organ metastases (HR 1.672; 95\% CI,
$1.059-2.641 ; P=0.027)$, and the levels of TCTCs (HR 2.133; 95\% CI, 1.253-3.361; $P=0.005$ ), EMCTCs (HR 1.760; 95\% CI, $1.012-3.060 ; P=0.045$ ), and MCTCs (HR 2.473; $95 \%$ CI, $1.394-4.387 ; P=0.002$ ) were also assessed as potential risk factors. Significant parameters identified in univariate Cox regression analysis were further analyzed in multivariate

Table 2 CTCs and disease-related characteristics

\begin{tabular}{|c|c|c|c|c|c|}
\hline Characteristics & Number & TCTCs & ECTCs & EMCTCs & MCTCs \\
\hline \multicolumn{6}{|l|}{ Pathologic type } \\
\hline Adenocarcinoma & 26 & $5.5(1.75,12.25)$ & $0.5(0,2)$ & $4(0,8.25)$ & $0(0,1.25)$ \\
\hline Adenosquamous & 3 & $8(2,10)$ & $\mathrm{I}(0, \mathrm{I})$ & $6(1,8)$ & $0(0,3)$ \\
\hline \multicolumn{6}{|l|}{ Location } \\
\hline Right lung & 19 & $7(I, 8)$ & $0(0,1)$ & $2(0,8)$ & $0(0,1)$ \\
\hline Left lung & 12 & $5.5(3.25,13)$ & I $(0,3.5)$ & $4.5(0.75,9.75)$ & $0(0,2.75)$ \\
\hline \multicolumn{6}{|l|}{ Tumor stage } \\
\hline III & 5 & I $(0,7)$ & $0(0,0)^{*}$ & $\mathrm{I}(0,7)$ & $0(0,0)$ \\
\hline IV & 24 & $7(3,12.75)$ & I $(0,2)^{*}$ & $4(1,8.75)$ & $0(0,2)$ \\
\hline \multicolumn{6}{|l|}{$\mathrm{T}$} \\
\hline 1 & 2 & $4(1,7)$ & $3(1,5)$ & I $(0,2)$ & $0(0,0)$ \\
\hline 2 & 2 & $6.5(3,10)$ & $0.5(0, I)$ & $4(2,6)$ & $2(1,3)$ \\
\hline 3 & 5 & $2(0,27)$ & $0(0,16)$ & I $(0,10)$ & $0(0,1.5)$ \\
\hline 4 & 20 & $7.5(3.25,12.75)$ & $0.5(0,2)$ & $4.5(I, 8.75)$ & $0(0,1.75)$ \\
\hline \multicolumn{6}{|c|}{ (1) } \\
\hline MO & 5 & I $(0,7)$ & $0(0,0)^{*}$ & I $(0,7)$ & $0(0,0)$ \\
\hline Mla & 2 & $6.5(0,13)$ & $0(0,0)^{*}$ & $5.5(0,11)$ & I $(0,2)$ \\
\hline MIb & 1 & 1 & 1 & 1 & 1 \\
\hline MIc & 22 & $7(3,12.5)$ & I $(0,2.5)^{*}$ & $4(1,8.25)$ & $0(0,2.25)$ \\
\hline \multicolumn{6}{|l|}{$\mathrm{N}$} \\
\hline No & 2 & $3.5(0,7)$ & I $(0,2)^{*}$ & $2(0,4)$ & $0.5(0,1)$ \\
\hline NI & 0 & 1 & 1 & 1 & I \\
\hline N2 & 10 & $3.5(0,8.5)$ & $0(0,0.25)^{*}$ & $3(0,6.5)$ & $0(0,3.25)$ \\
\hline N3 & 17 & $7(3.5,13.5)$ & I $(0,4.5)^{*}$ & $6(1,11)$ & $0(I, I .5)$ \\
\hline
\end{tabular}

Notes: $* P<0.05$; statistical description: median (figures outside the parentheses) and interquartile range (figures in the parentheses).

Abbreviations: CTCs, circulating tumor cells; ECTCs, epithelial circulating tumor cells; EMCTCs, epithelial-mesenchymal circulating tumor cells; MCTCs, mesenchymal circulating tumor cells; TCTCs, total CTCs. 
Table 3 CTCs and organ metastases

\begin{tabular}{|c|c|c|c|c|c|}
\hline Characteristics & Number & TCTCs & ECTCs & EMCTCs & MCTCs \\
\hline \multicolumn{6}{|c|}{ Medium or large pleural effusion } \\
\hline Yes & 7 & $8(2,30)$ & $I(0,6)$ & $4(1,14)$ & $3(0,4)^{*}$ \\
\hline No & 23 & $5(1,8)$ & $0(0,2)$ & $4(0,8)$ & $0(0,0)^{*}$ \\
\hline \multicolumn{6}{|c|}{ Intra-lung metastases } \\
\hline Yes & 24 & $5.5(2,12.75)$ & $I(0,2)$ & $4.5(0.25,8.75)$ & $0(0,1)$ \\
\hline No & 5 & $3(0.5,8.5)$ & $I(0,3)$ & $2(0,4)$ & $0(0,2)$ \\
\hline \multicolumn{6}{|c|}{ Homolateral lung metastases } \\
\hline Yes & 23 & $6(2,13)$ & $I(0,2)$ & $5(I, 9)$ & $0(0,1)$ \\
\hline No & 6 & $2.5(0.75,7.75)$ & $\mathrm{I}(0,2.75)$ & $I(0,3)$ & $0(0,1.5)$ \\
\hline \multicolumn{6}{|c|}{ Contralateral lung metastases } \\
\hline Yes & 15 & $7(3,14)$ & $I(0,2)$ & $6(0,11)$ & $0(0,2)$ \\
\hline No & 14 & $3.5(1,7.25)$ & $0(0,1)$ & $1.5(0,6)$ & $0(0,0.25)$ \\
\hline \multicolumn{6}{|c|}{ Extra-lung metastases } \\
\hline Yes & 22 & $7(3,12.5)$ & $\mathrm{I}(0,2.5)^{*}$ & $4(I, 8.25)$ & $0(0,2.25)$ \\
\hline No & 7 & I $(0,8)$ & $0(0,0)^{*}$ & I $(0,8)$ & $0(0,0)$ \\
\hline \multicolumn{6}{|l|}{ Bone metastases } \\
\hline Yes & 20 & $7(3.25,13.5)$ & $\mathrm{I}(0,3.5)^{*}$ & $4(1.25,8.75)$ & $0(0,2.75)$ \\
\hline No & 9 & I $(0,7.5)$ & $0(0,0.5)^{*}$ & I $(0,7)$ & $0(0,0)$ \\
\hline \multicolumn{6}{|c|}{ Cranium metastases } \\
\hline Yes & 4 & $5(2,24.5)$ & I $(0.25,4.75)$ & $2.5(1,13)$ & $2(0,7)$ \\
\hline No & 25 & $6(2,11)$ & $0(0,2)$ & $4(0,8)$ & $0(0,1)$ \\
\hline \multicolumn{6}{|c|}{ Truncal bone (except vertebra) metastases } \\
\hline Yes & 10 & $5(2.75,30.5)$ & $\mathrm{I}(0,5.25)$ & $3.5(0.75,9.5)$ & $0.5(0,4.25)$ \\
\hline No & 19 & $7(I, 8)$ & $0(0, I)$ & $4(0,8)$ & $0(0,1)$ \\
\hline \multicolumn{6}{|c|}{ Vertebra metastases } \\
\hline Yes & 15 & $7(3,14)$ & $\mathrm{I}(0,2)^{*}$ & $4(1,9)$ & $0(0,2)$ \\
\hline No & 14 & $5(0.75,8)$ & $0(0,1)^{*}$ & $3.5(0,6.5)$ & $0(0,0.5)$ \\
\hline \multicolumn{6}{|c|}{ Extremities metastases } \\
\hline Yes & 6 & $3.5(2.75,11.25)$ & $0.5(0,4.5)$ & $2.5(0.75,7.75)$ & $0(0,2.75)$ \\
\hline No & 23 & $7(1,12)$ & $\mathrm{I}(0,2)$ & $4(0,8)$ & $0(0,2)$ \\
\hline \multicolumn{6}{|c|}{ Abdominal metastases } \\
\hline Yes & 7 & $5(2,32)$ & $I(0,6)$ & $5(1,14)$ & $0(0,8)$ \\
\hline No & 22 & $6.5(1.75,8.5)$ & $0.5(0,2)$ & $3.5(0,6.5)$ & $0(0,1)$ \\
\hline \multicolumn{6}{|l|}{ Kidney metastases } \\
\hline Yes & 3 & $5(1,30)$ & $I(0,6)$ & $5(0,16)$ & $0(0,8)$ \\
\hline No & 26 & $6.5(2,10.5)$ & $0.5(0,2)$ & $4(0.75,8)$ & $0(0,1.25)$ \\
\hline \multicolumn{6}{|c|}{ Adrenal gland metastases } \\
\hline Yes & 4 & $18(2.5,44)$ & $0.5(0,23.5)$ & $6(1.75,12.5)$ & $1.5(0,18.75)$ \\
\hline No & 25 & $6(1.5,9)$ & $I(0,2)$ & $4(0,7)$ & $0(0,1)$ \\
\hline \multicolumn{6}{|l|}{ Liver metastases } \\
\hline Yes & 1 & $2(2,2)$ & $\mathrm{I}(\mathrm{I}, \mathrm{I})$ & $\mathrm{I}(\mathrm{I}, \mathrm{I})$ & $0(0,0)$ \\
\hline No & 28 & $6.5(2.25,11.5)$ & $0.5(0,2)$ & $4(0.25,8)$ & $0(0,1.75)$ \\
\hline \multicolumn{6}{|l|}{ Brain metastases } \\
\hline Yes & 10 & $9(6.5,15.25)$ & I $(0.75,2.75)$ & $4(3.5,11.75)$ & $0.5(0,3)$ \\
\hline No & 19 & $4(1,8)$ & $0(0,2)$ & $2(0,6)$ & $0(0, I)$ \\
\hline \multicolumn{6}{|c|}{ Number of organ metastases } \\
\hline$<3$ & 17 & $5(0.5,7.5)^{*}$ & $0(0,1.5)$ & $3(0,6)^{*}$ & $0(0,0)^{*}$ \\
\hline$\geq 3$ & 12 & II $(3.25,27.25)^{*}$ & I $(0.25,3.5)$ & $7(1.25,13.25)^{*}$ & I $(0,3)^{*}$ \\
\hline
\end{tabular}

Notes: $* P<0.05$; statistical description: median (figures outside the parentheses) and interquartile range (figures in the parentheses).

Abbreviations: CTCs, circulating tumor cells; ECTCs, epithelial circulating tumor cells; EMCTCs, epithelial-mesenchymal circulating tumor cells; MCTCs, mesenchymal circulating tumor cells; TCTCs, total CTCs.

Cox regression analysis. The results showed that the levels of EMCTCs (HR 2.204; 95\% CI, 1.099-4.420; $P=0.026$ ) and MCTCs (HR 2.308; 95\% CI, 1.166-4.569; $P=0.016$ ) were associated with worse clinical outcomes (Table 4). The results were confirmed by Kaplan-Meier (Figure 4).

\section{Correlation between CTCs and peripheral T cells}

The results showed that the levels of TCTCs, ECTCs, and MCTCs were negatively associated with the levels of $\mathrm{CD}^{+}$ $\mathrm{T}$ cells, $\mathrm{CD}^{+} \mathrm{T}$ cells, or $\mathrm{CD} 8^{+}$(cytotoxic) $\mathrm{T}$ cells, however 
Table 4 Cox regression of overall survival

\begin{tabular}{|c|c|c|c|c|c|c|}
\hline & \multicolumn{2}{|c|}{ Univariate Cox regression } & \multirow[t]{2}{*}{$P$-value } & \multicolumn{2}{|c|}{ Multivariate Cox regression } & \multirow[t]{2}{*}{$P$-value } \\
\hline & HR & $95 \% \mathrm{Cl}$ & & HR & $95 \% \mathrm{Cl}$ & \\
\hline Gender $(\mathrm{F}=\mathrm{I})$ & 1.025 & $(0.3 \mid 2,3.362)$ & 0.968 & - & - & - \\
\hline Age $(\geq 65=1)$ & 3.929 & $(1.190,12.971)$ & $0.027 *$ & - & - & - \\
\hline Smoking & 0.987 & $(0.289,3.373)$ & 0.983 & - & - & - \\
\hline Tumor history & 0.891 & $(0.114,6.966)$ & 0.912 & - & - & - \\
\hline Pathologic type & 0.357 & $(0.074,1.723)$ & 0.200 & - & - & - \\
\hline Lump location & 0.708 & $(0.355, \mathrm{I} .4 \mathrm{II})$ & 0.326 & - & - & - \\
\hline Number of organ metastases & 1.672 & $(1.059,2.641)$ & $0.027 *$ & - & - & - \\
\hline T stage & 1.141 & $(0.517,2.518)$ & 0.744 & - & - & - \\
\hline $\mathrm{N}$ stage & 1.179 & $(0.496,4.808)$ & 0.709 & - & - & - \\
\hline M stage & 3.686 & $(0.377,36.058)$ & 0.262 & - & - & - \\
\hline TCTCs & 2.133 & $(1.253,3.631)^{\prime}$ & $0.005^{*}$ & - & - & - \\
\hline \multicolumn{7}{|l|}{$[0,6]$} \\
\hline \multicolumn{7}{|l|}{$[7,13]$} \\
\hline \multicolumn{7}{|l|}{$[14,20]$} \\
\hline \multicolumn{7}{|l|}{$[21,+\infty]$} \\
\hline ECTCs & 1.314 & $(0.636,2.717)$ & 0.461 & - & - & - \\
\hline \multicolumn{7}{|l|}{$[0,3]$} \\
\hline \multicolumn{7}{|l|}{$[4,7]$} \\
\hline \multicolumn{7}{|l|}{$[8, \mathrm{II}]$} \\
\hline \multicolumn{7}{|l|}{$[12,+\infty)$} \\
\hline EMCTCs & 1.760 & $(1.012,3.060)$ & $0.045^{*}$ & 2.204 & $(1.099,4.420)$ & $0.026^{*}$ \\
\hline \multicolumn{7}{|l|}{$[0,3]$} \\
\hline \multicolumn{7}{|l|}{$[4,7]$} \\
\hline \multicolumn{7}{|l|}{$[8,1 \mathrm{I}]$} \\
\hline \multicolumn{7}{|l|}{$[12,+\infty)$} \\
\hline MCTCs & 2.473 & $(1.394,4.387)$ & $0.002 *$ & 2.308 & $(1.166,4.569)$ & $0.016^{*}$ \\
\hline \multicolumn{7}{|l|}{$[0,3]$} \\
\hline \multicolumn{7}{|l|}{$[4,7]$} \\
\hline \multicolumn{7}{|l|}{$[8,11]$} \\
\hline \multicolumn{7}{|l|}{$[12,+\infty)$} \\
\hline Mutation + target therapy & 0.172 & $(0.022,1.342)$ & 0.093 & - & - & - \\
\hline
\end{tabular}

Note: $* P<0.05$

Abbreviations: $\mathrm{Cl}$, confidence index; CTCs, circulating tumor cells; ECTCs, epithelial circulating tumor cells; EMCTCs, epithelial-mesenchymal circulating tumor cells; MCTCs, mesenchymal circulating tumor cells; TCTCs, total CTCs.
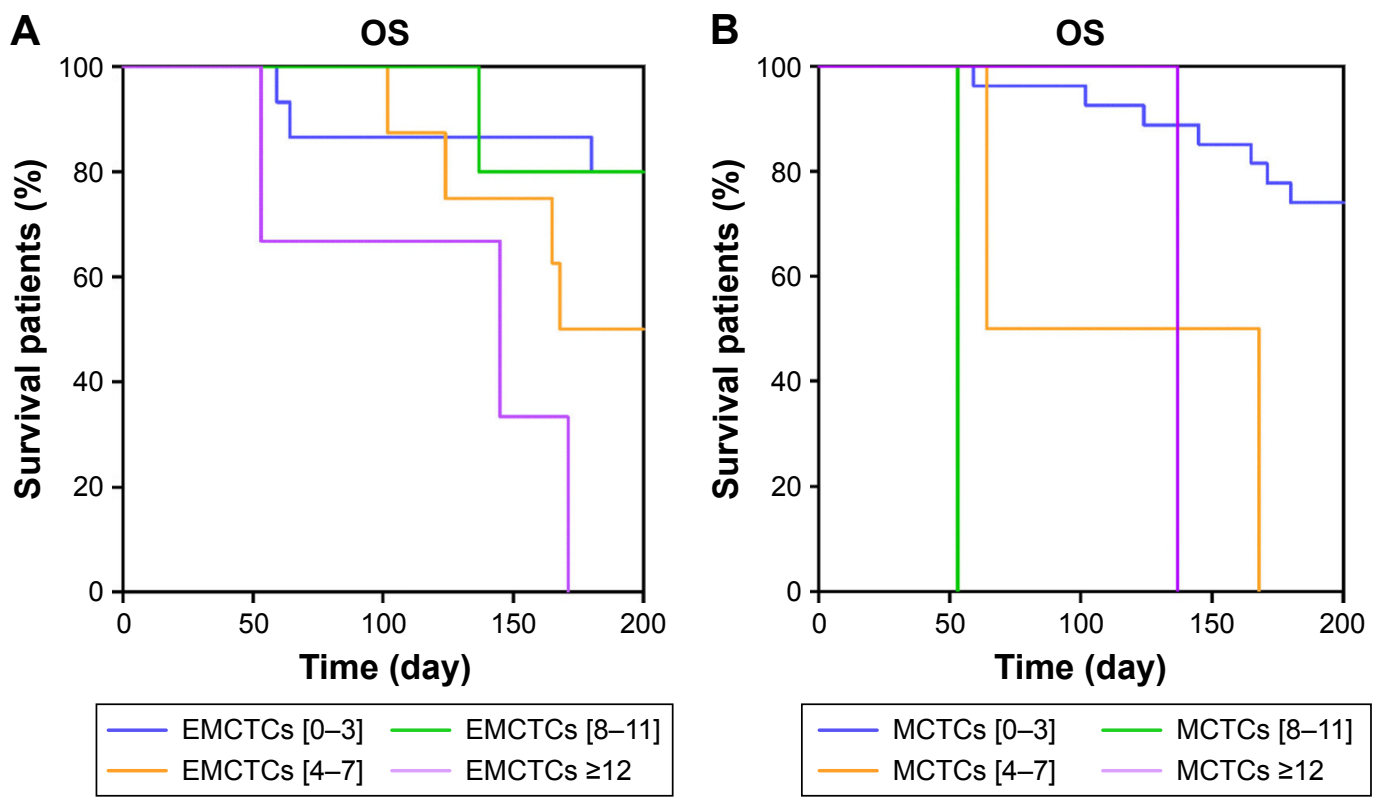

Figure 4 (A) Kaplan-Meier life-table analysis showed EMCTCs were associated with worsened clinical outcomes. (B) Kaplan-Meier life-table analysis showed MCTCs had negative prognostic value.

Abbreviations: EMCTCs, epithelial-mesenchymal circulating tumor cells; MCTCs, mesenchymal circulating tumor cells; OS, overall survival. 
Table 5 Correlations of T-cell-related CD and CTCs

\begin{tabular}{lll}
\hline & $\boldsymbol{r}$ & $\boldsymbol{P}$-value \\
\hline CD3 $^{+}$ & & \\
TCTC & -0.321 & 0.264 \\
ECTC & -0.097 & 0.749 \\
EMCTC & -0.659 & $0.01^{*}$ \\
MCTC & -0.256 & 0.376 \\
CD4 & & \\
TCTC & & 0.716 \\
ECTC & -0.107 & 0.846 \\
EMCTC & -0.057 & 0.409 \\
MCTC & -0.24 & 0.948 \\
CD8 & -0.019 & \\
TCTC & & 0.163 \\
ECTC & -0.394 & 0.458 \\
EMCTC & -0.216 & $0.04^{*}$ \\
MCTC & -0.713 & 0.366 \\
\hline
\end{tabular}

Note: $* P<0.05$.

Abbreviations: $C D$, cluster of differentiation; CTCs, circulating tumor cells; ECTCs, epithelial circulating tumor cells; EMCTCs, epithelial-mesenchymal circulating tumor cells; MCTCs, mesenchymal circulating tumor cells; TCTCs, total CTCs.

the associations were insignificant. However, the levels of EMCTCs were significantly associated with the levels of $\mathrm{CD}^{+} \mathrm{T}$ cells $(r=-0.659 ; P=0.01)$ and those of cytotoxic T cells $\left(C D 8^{+} ; r=-0.713 ; P=0.04\right)$, but were not significantly associated with the levels of $\mathrm{CD}^{+} \mathrm{T}$ cells (Table 5).

\section{Discussion}

Our study focused on exploring the association of CTCs, particularly subpopulations of CTCs, with the clinicopathologic characteristics including metastases and prognosis. The association between CTCs and T cells should be investigated, as $\mathrm{T}$ cells play an important role in antitumor microenvironment.

CTC subpopulations were detected based on the presence of epithelial and mesenchymal markers. In addition to EpCAMs, CK 8/18/19, which were used as epithelial markers in this CanPatrol CTC enrichment system, E-cadherin, mucin-1, claudins 3, 4, and 7, plakoglobin, and desmoplakin were also regarded as epithelial markers. ${ }^{16}$ Fibronectin, $\mathrm{N}$-cadherin, actin 2 , nuclear catenin $\beta 1$, and fibroblast growth factor 2 were the other mesenchymal markers, besides vimentin and Twist. ${ }^{16}$ The process of epithelial-mesenchymal transition (EMT) refers to the loss of epithelial markers and the expression of mesenchymal markers. ${ }^{16}$

In this study, EMCTCs predominated among the three subpopulations of CTCs in 16 patients. Hanssen et a $1^{17}$ found that the usage of multiple cell surface proteins for CTC capture and isolation in NSCLC patients increased the sensitivity for CTC detection. Although the clinical study of Hanssen et $a{ }^{17}$ did not examine EMCTCs, it emphasized the significance of phenotyping CTCs in clinical detection. Millions of cancer cells disseminate from the solid primary or secondary lesions, but only a few of the CTCs can reach the remote organs and form metastases. Several laboratory studies found that EMCTCs could migrate in order to invade more effectively. ${ }^{18,19}$ EMT can inhibit the apoptosis of CTCs and makes CTCs difficult to be identified in hematopoietic cell microenvironment, thereby allowing evasion from the immune system. ${ }^{20}$

Nevertheless, phenotype transformation is not always required for the migration of tumor cells. ${ }^{21-23}$ The mesenchymal phenotype of CTCs that undergo EMT promotes the motility of CTCs, but does not favor growth. Indeed, cancer cells must undergo the mesenchymal-epithelial transition to acquire the ability of proliferation. ${ }^{24}$ EMCTCs undergo partial EMT. Therefore, compared with ECTCs, EMCTCs are more invasive, and compared with MCTCs, EMCTCs are more proliferative. Hence, EMCTCs predominating within the CTC subpopulations could accelerate the invasiveness of the tumor and the formation of metastases.

Our study found that the levels of ECTCs were positively correlated with tumor stage and $\mathrm{M}$ stage. A meta-analysis of 1,576 NSCLC patients, conducted by Wang et al, ${ }^{9}$ also showed that the number of CTCs is positively correlated with tumor stage and lymph node metastasis, but not significantly associated with histology. Furthermore, Wan et al ${ }^{10}$ showed that CTC levels are not significantly correlated with gender, age, or pathologic type $(P>0.05)$, but are closely related with clinical stage $(P<0.01)$ and tumor progression. However, we found that the ECTC levels of N0 patients were greater than those of N2 patients and the levels of ECTCs were positively correlated with age; the reason for which may be the small size of the sample. Also, TCTC levels of patients who had undergone treatment were significantly higher than those of untreated patients. The reasons for the above results are as follows. Firstly, the treated patients received improper therapeutics before being treated in Ruijin Hospital; secondly, the treated patients did not strictly follow the doctors' orders; thirdly, all the treated patients belonged to tumor stage IV. The first two reasons could lead to progression of the disease.

As the extent of tumor cell dissemination and impairment of different organs can reflect the progression of lung cancer, it is crucial to assess the latent association between the levels of CTCs and organ metastases. ${ }^{20}$ In our study, the levels of TCTCs, EMCTCs, and MCTCs showed statistical differences in the number of organ metastases. Additionally, MCTC levels were positively correlated with the severity of malignant pleural effusion, which may indicate that the 
levels of MCTCs may reflect the clinical characteristics and that TCTC levels may not.

In this study, bone was found to be the most common homing site for CTCs. Twenty-seven patients were detected with CTCs; 19 of these 27 patients had bone metastases. Therefore, bone marrow may function as a reservoir for disseminated tumor cells (DTCs). Pantel and Brakenhoff ${ }^{25}$ and Riethdorf et $\mathrm{al}^{26}$ found that bone was the most favorable homing site and reservoir for CTCs and DTCs, even though other organs such as lung and brain can serve as storage for DTCs which were not discovered as easily as DTCs in the bone marrow. DTCs in these reservoirs can stay dormant, thereby escaping systemic therapy and immunologic attack. ${ }^{27}$

Different parts of bones have different potentials to form metastases. Vertebrae were more likely to form metastases, which demonstrated that different bones possess different microenvironments where CTCs are inclined to settle in. DTCs could still disseminate to other remote organs even the primary tumor has been resected for years. ${ }^{23}$ Bone metastases can be applied as the prognostic factor to colon cancer with rare occurrence of bone metastases, as well as to breast cancer with frequent occurrence of bone metastases. ${ }^{28}$ Thus, further studies should focus on promoting the diagnosis of bone metastases and developing more effective therapeutics to prevent and improve bone metastases.

Our study showed that EMCTCs and MCTCs had association with worse clinical outcomes. TCTCs, however, had no significant correlation with OS. Presently, few studies explore the correlation between the subpopulations of CTCs and OS. In their research of lung cancer, Punnoose et $\mathrm{al}^{11}$ found that higher baseline CTC levels and decreased CTC levels upon treatment were associated with the response assessed by the Response Evaluation Criteria in Solid Tumors and longer progression-free survival. CTC levels are also regarded as a prognostic marker of metastatic prostate, breast, and colorectal cancer. ${ }^{12,29,30}$ Although the above studies indicate that CTCs can be used as a prognostic factor, Hanssen et $\mathrm{al}^{17}$ had emphasized the significance of analyzing the subpopulations of CTCs. The results of our study, to some extent, indicated the prognostic values of the CTC subpopulations. However, due to the small sample size of our study, it is too early to conclude that EMCTCs and MCTCs were the independent prognostic factors. Studies with larger sample size of NSCLC patients are needed to further investigate the prognostic values of the CTC subpopulations.

$T$ cells play a crucial role in defending against infection and cancer. ${ }^{5,6}$ Nevertheless, few clinical studies have investigated the association between CTCs and T cells. We found that T-cell levels were negatively associated with the levels of CTCs no matter whether these correlations were significant or not. Furthermore, EMCTCs were negatively and significantly associated with $\mathrm{CD}^{+} \mathrm{T}$ cells and cytotoxic $\left(\mathrm{CD} 8^{+}\right)$ T cells, which, to some extent, corresponded to the studies by Mego et $\mathrm{al}^{7}$ and Gruber et al. ${ }^{5}$ Mego et $\mathrm{al}^{7}$ pointed out that patients with $\geq 1$ CTC had lower percentage of T-cell receptor-activated $\mathrm{CD}^{+} \mathrm{T}$ cells and a higher percentage of T-regulatory lymphocytes, compared to patients without CTCs. Gruber et $\mathrm{al}^{5}$ found that in breast cancer, $\mathrm{CD}^{+}$ $\mathrm{T}$ cells and $\mathrm{CD} 8^{+} \mathrm{T}$ cells showed no significant association with CTCs, but CD95 (FAS)-positive T-helper cells were significantly increased in CTC-positive cases. Several studies indicated that a strong infiltration of cytotoxic $\mathrm{T}$ cells correlates with longer survival. ${ }^{31,32}$ Hence, impairment of the immune system can lead to the occurrence of increasing peripheral CTCs. Energy deficiency and glycolysis which is due to hypoxia leads to restraint of activation, proliferation, and longevity of $\mathrm{CD}^{+} \mathrm{T}$ cells.${ }^{33-35}$ Hence, the levels of CTCs were negatively correlated with $\mathrm{CD}^{+} \mathrm{T}$-cell-mediated immune response.

However, in the detection of $\mathrm{T}$ cells, we were unable to classify $\mathrm{CD}^{+} \mathrm{T}$ cells into $\mathrm{T}$-helper cells and T-regulatory cells. In our study, the levels of $\mathrm{CD}^{+} \mathrm{T}$ cells were negatively associated with CTCs, however the results were insignificant. As the components of $\mathrm{CD}^{+} \mathrm{T}$ cells are complex, different types of $\mathrm{CD}^{+} \mathrm{T}$ cells may have reverse association with survival or the levels of CTCs. For example, a study by Tosolini et $\mathrm{al}^{36}$ discovered that in colorectal cancer, infiltrating Th1 cells and $\mathrm{CD}^{+}$cytotoxic $\mathrm{T}$ cells constituted a positive prognostic sign; by contrast, myeloid cells and the expression of Th17 cells were negatively associated with disease-free survival.

\section{Conclusion}

Analyzing the molecular characteristics of CTCs is crucial to understand and inhibit the biologic characteristics of tumor metastasis. The mechanism of the behaviors of the CTC subpopulations in metastasis, prognosis, and the immune system is still unclear. Whether EMT is a necessity and what kind of microenvironment in the bone marrow drives CTC homing preference, proliferation, and remetastasizing needs further investigation. In addition, it is still a challenge to apply the correlations of CTC subpopulations and cancer immunoediting mediated by $\mathrm{T}$ cells to diagnose the severity of cancer and to conduct therapeutics. Clinical studies with larger sample and longer periods of follow-up are needed to validate these findings. 


\section{Acknowledgments}

The authors gratefully acknowledge the participants and the doctors who helped them.

This study is supported by Shanghai Municipal Commission of Health and Family Planning for Key Discipline Establishment (2015ZB0503) and Charity Projects. ZhiHong $\mathrm{Xu}$ is the co-first author.

\section{Disclosure}

The authors report no conflicts of interest in this work.

\section{References}

1. Ferlay J, Soerjomataram I, Dikshit R, et al. Cancer incidence and mortality worldwide: sources, methods and major patterns in GLOBOCAN 2012. Int J Cancer. 2015;136(5):E359-E386.

2. Nguyen DX, Bos PD, Massague J. Metastasis: from dissemination to organ-specific colonization. Nat Rev Cancer. 2009;9(4):274-284.

3. Pantel K, Alix-Panabières C. Real-time liquid biopsy in cancer patients: fact or fiction? Cancer Res. 2013;73(21):6384-6388.

4. Yu M, Bardia A, Wittner BS, et al. Circulating breast tumor cells exhibit dynamic changes in epithelial and mesenchymal composition. Science. 2013;339(6119):580-584.

5. Gruber I, Landenberger N, Staebler A, Hahn M, Wallwiener D, Fehm T. Relationship between circulating tumor cells and peripheral T-cells in patients with primary breast cancer. Anticancer Res. 2013;33(5): 2233-2238.

6. Clever D, Roychoudhuri R, Constantinides MG, et al. Oxygen sensing by $\mathrm{T}$ cells establishes an immunologically tolerant metastatic niche. Cell. 2016;166(5):1117-1131.

7. Mego M, Gao H, Cohen EN, et al. Circulating tumor cells (CTC) are associated with defects in adaptive immunity in patients with inflammatory breast cancer. J Cancer. 2016;7(9):1095-1104.

8. Mittal D, Gubin MM, Schreiber RD, Smyth MJ. New insights into cancer immunoediting and its three component phases-elimination, equilibrium and escape. Curr Opin Immunol. 2014;27:6-25.

9. Wang J, Wang K, Xu J, Huang J, Zhang T. Prognostic significance of circulating tumor cells in non-small-cell lung cancer patients: a metaanalysis. PLoS One. 2013;8(11):e78070.

10. Wan JW, Gao MZ, Hu RJ, et al. A preliminary study on the relationship between circulating tumor cells count and clinical features in patients with non-small cell lung cancer. Ann Transl Med. 2015;3(22):352.

11. Punnoose EA, Atwal S, Liu W, et al. Evaluation of circulating tumor cells and circulating tumor DNA in non-small cell lung cancer: association with clinical endpoints in a phase II clinical trial of pertuzumab and erlotinib. Clin Cancer Res. 2012;18(8):2391-2401.

12. Thalgott M, Rack B, Eiber M, et al. Categorical versus continuous circulating tumor cell enumeration as early surrogate marker for therapy response and prognosis during docetaxel therapy in metastatic prostate cancer patients. BMC Cancer. 2015;15:458.

13. Goldstraw P, Chansky K, Crowley J, et al. The IASLC lung cancer staging project: proposals for revision of the TNM stage groupings in the forthcoming (Eighth) edition of the TNM classification for lung cancer. J Thorac Oncol. 2016;11(1):39-51.

14. Tsongalis GJ. Branched DNA technology in molecular diagnostics. Am $J$ Clin Pathol. 2006;126(3):448-453.

15. Wu S, Liu S, Liu Z, et al. Classification of circulating tumor cells by epithelial-mesenchymal transition markers. PLoS One. 2015;10(4): e0123976.
16. Bednarz-Knoll N, Alix-Panabières C, Pantel K. Plasticity of disseminating cancer cells in patients with epithelial malignancies. Cancer Metastasis Rev. 2012;31(3-4):673-687.

17. Hanssen A, Wagner J, Gorges TM, et al. Characterization of different CTC subpopulations in non-small cell lung cancer. Sci Rep. 2016;6:28010.

18. Barriere G, Riouallon A, Renaudie J, Tartary M, Rigaud M. Mesenchymal characterization: alternative to simple CTC detection in two clinical trials. Anticancer Res. 2012;32(8):3363-3369.

19. Tam WL, Weinberg RA. The epigenetics of epithelial-mesenchymal plasticity in cancer. Nat Med. 2013;19(11):1438-1449.

20. Joosse SA, Gorges TM, Pantel K. Biology, detection, and clinical implications of circulating tumor cells. EMBO Mol Med. 2014;7(1):1-11.

21. Godinho SA, Picone R, Burute M, et al. Oncogene-like induction of cellular invasion from centrosome amplification. Nature. 2014; 510(7503):167-171.

22. Aceto N, Bardia A, Miyamoto DT, et al. Circulating tumor cell clusters are oligoclonal precursors of breast cancer metastasis. Cell. 2014; 158(5):1110-1122.

23. Förnvik D, Andersson I, Dustler M, et al. No evidence for shedding of circulating tumor cells to the peripheral venous blood as a result of mammographic breast compression. Breast Cancer Res Treat. 2013; 141(2):187-195.

24. Celià-Terrassa T, Meca-Cortés O, Mateo F, et al. Epithelial-mesenchymal transition can suppress major attributes of human epithelial tumorinitiating cells. J Clin Invest. 2012;122(5):1849-1868.

25. Pantel K, Brakenhoff RH. Dissecting the metastatic cascade. Nat Rev Cancer. 2004;4(6):448-456.

26. Riethdorf S, Wikman H, Pantel K. Review: biological relevance of disseminated tumor cells in cancer patients. Int J Cancer. 2008;123(9): 1991-2006.

27. Kang Y, Pantel K. Tumor cell dissemination: emerging biological insights from animal models and cancer patients. Cancer Cell. 2013;23(5):573-581.

28. Pantel K, Alix-Panabières C. Bone marrow as a reservoir for disseminated tumor cells: a special source for liquid biopsy in cancer patients. Bonekey Rep. 2014;3:584.

29. Bidard FC, Peeters DJ, Fehm T, et al. Clinical validity of circulating tumour cells in patients with metastatic breast cancer: a pooled analysis of individual patient data. Lancet Oncol. 2014;15(4):406-414.

30. Krebs MG, Renehan AG, Backen A, et al. Circulating tumor cell enumeration in a phase II trial of a four-drug regimen in advanced colorectal cancer. Clin Colorectal Cancer. 2015;14(2):115-122.

31. Kitamura T, Qian BZ, Pollard JW. Immune cell promotion of metastasis. Nat Rev Immunol. 2015;15(2):73-86.

32. Pancione M, Giordano G, Remo A, et al. Immune escape mechanisms in colorectal cancer pathogenesis and liver metastasis. J Immunol Res. 2014;2014:686879.

33. Larbi A, Zelba H, Goldeck D, Pawelec G. Induction of HIF-1alpha and the glycolytic pathway alters apoptotic and differentiation profiles of activated human T cells. J Leukoc Biol. 2010;87(2):265-273.

34. Wang R, Green DR. Metabolic checkpoints in activated T cells. Nat Immunol. 2012;13(10):907-915.

35. Chang CH, Qiu J, O'Sullivan D, et al. Metabolic competition in the tumor microenvironment is a driver of cancer progression. Cell. 2015;162(6): 1229-1241.

36. Tosolini M, Kirilovsky A, Mlecnik B, et al. Clinical impact of different classes of infiltrating T cytotoxic and helper cells (Th1, th2, treg, th17) in patients with colorectal cancer. Cancer Res. 2011;71(4):1263-1271. 


\section{Publish your work in this journal}

OncoTargets and Therapy is an international, peer-reviewed, open access journal focusing on the pathological basis of all cancers, potential targets for therapy and treatment protocols employed to improve the management of cancer patients. The journal also focuses on the impact of management programs and new therapeutic agents and protocols on

patient perspectives such as quality of life, adherence and satisfaction. The manuscript management system is completely online and includes a very quick and fair peer-review system, which is all easy to use. Visit http://www.dovepress.com/testimonials.php to read real quotes from published authors.

Submit your manuscript here: http://www.dovepress.com/oncotargets-and-therapy-journal 